\title{
MULTIVALUED OPERATIONS AND UNIVERSAL COALGEBRA
}

\author{
ROBERT C. DAVIS
}

\begin{abstract}
We define a type of representation of a semigroup by relations on a set, more general than the representation by transformations, which leads to a category cotripleable over the category of sets. This result motivates a generalization to higherorder operations and a concept of cotheory resembling that of theory in universal algebra.
\end{abstract}

1. Introduction. The representation of a semigroup $M$ by transformations of a set $A$ amounts to a homomorphism of $M$ into the semigroup of functions $A \rightarrow A$. The (left) $M$-sets are the objects of a category which is cotripleable over the category $\mathscr{S}$ of sets, the right adjoint $F$ being given by $F A=A \times A^{M}$ for each set $A$. Here $M$ acts on $F A$ by $m(a, h)=(h(m), m h)$ where $(m h)(n)=h(n m)$. In particular, $F A$ is universal in that any representation of $M$ on $A$ is embeddable in $F A$.

The relations on $A$ also form a semigroup $\mathscr{R}(A)$, whose elements can be thought of as functions $m: A \rightarrow P A=$ power set of $A$. Composition is defined by $(m n) a=\bigcup\left\{m a^{\prime} \mid a^{\prime} \in n a\right\}$. We can also represent $M$ by relations on $A$, i.e. by a homomorphism $M \rightarrow \mathscr{R}(A)$. The category of $M$-sets in this sense does not admit a right adjoint, in general; this may be proved as in [2].

In this paper we exhibit a notion of representation lying between these two in generality which yields a cotripleable category. This will appear fortunate in view of the fact that the new kind of representation is not equivalent to a homomorphism from $M$ into any fixed semigroup connected with $A$.

The cotripleable categories so obtained admit an immediate generalization to categories of algebras over certain "cotheories" roughly analogous to the theories of universal algebra [4]. As noted in [2], the results of [1] imply that universal coalgebra has a notion of theory which is in principle exactly analogous to the one in universal algebra, but in practice is

Received by the editors April 15, 1971.

AMS 1970 subject classifications. Primary 08A25, 08C15; Secondary 20M99.

Key words and phrases. Semigroup, representation, cotripleable category, right adjoint, colimit, right complete category, cosolution set, precise cotripleableness condition, tractable standard cotheory.

(C) American Mathematical Society 1972 
awkward. This fact prompts a search for other characterizations of cotripleable categories. The concept introduced here appears to point in a promising direction.

2. Multivalued $M$-sets. If a semigroup $M$ acts on a set $A$ in the usual sense then $m(n a)=(m n) a$. One way to interpret this equation when $M$ is represented by many-valued functions (relations) is that $m a^{\prime}=(m n) a$ for each $a^{\prime}$ in $n a$; $(m n) a$ would be empty if $n a$ is. A representation satisfying this requirement will be called a multivalued representation, or $\mu$-representation. A relation $R$ on $A$ can be in the image of a $\mu$-representation of some $M$ iff $R(a, b)$ and $R(a, c)$ imply that $\{x \mid R(b, x)\}=\{x \mid R(c, x)\}$; for, if $R$ satisfies this condition, the cyclic subsemigroup of $\mathscr{R}(A)$ generated by $R$ will be $\mu$-represented. (Here, as usual, $R \subseteq A \times A$ corresponds to $m: A \rightarrow P A$ via $b \in m a$ iff $R(a, b)$.) A relation $R$ satisfying this condition will be called a $\mu$-relation. However, the $\mu$-relations are not closed under composition and do not form a subsemigroup of $\mathscr{R}(A)$. Thus, for example, let $A=\{1,2,3,4\}, R=\{(1,2),(1,3),(2,4),(3,4)\}$ and $S=\{(2,1),(2,4)$, $(1,3),(4,3)\}$. Then $R$ and $S$ are $\mu$-relations but $R S=\{(1,4),(2,2),(2,3)$, $(4,4)\}$ is not a $\mu$-relation. In the face of this fact, the right adjoint $F$ to be introduced below provides a way to get hold of the $\mu$-representations: a $\mu$-representation of $M$ on $A$ is a subalgebra of $F A$.

If $M$ has a $\mu$-representation on both $A$ and $B$, a function $f: A \rightarrow B$ will be called a homomorphism if for each $a$ and $m, m f(a)=f(m a)$ (direct image). It is straightforward to check that the evident forgetful functor from the resulting category $\mathscr{M}$ to $\mathscr{S}$ satisfies the precise cotripleableness condition. This means that if $f, g: A \rightarrow B$ in $\mathscr{M}$ and we have in $\mathscr{S}$ a diagram

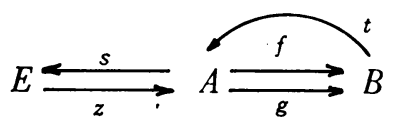

where $s z=1_{E}, t f=1_{A}, t g=z s$, and $f z=g z$, then there is a unique $\mu$-representation of $M$ on $E$ making $z$ a homomorphism, and $z$ is then the equalizer of $f$ and $g$ in $\mathscr{M}$. Hence $\mathscr{M}$ is cotripleable iff the forgetful functor has a right adjoint; see [1] or [3] for details.

In case $M$ is commutative we can exhibit the right adjoint $F: \mathscr{S} \rightarrow \mathscr{M}$ explicitly. For each set $A$ let $F A=A \times(P A)^{M}$ and define for each $n$ in $M$, $n\left(a,\left(\alpha_{m}\right)_{m \in M}\right)=\left\{\left(a^{\prime},\left(\alpha_{n m}\right)_{m \in M}\right) \mid a^{\prime} \in \alpha_{n}\right\}$. Then $F A$ is an object of $\mathscr{M}$, since

$$
\begin{aligned}
(r n)\left(a,\left(\alpha_{m}\right)_{m \in M}\right) & =\left\{\left(a^{\prime \prime},\left(\alpha_{r n m}\right)_{m \in M}\right) \mid a^{\prime \prime} \in \alpha_{r n}\right\} \\
& =\left\{\left(a^{\prime \prime},\left(\alpha_{r n m}\right)_{m \in M}\right) \mid a^{\prime \prime} \in \alpha_{n r}\right\} \\
& =r\left(a^{\prime},\left(\alpha_{n m}\right)_{m \in M}\right)
\end{aligned}
$$

for each $a^{\prime}$ in $\alpha_{n}$. Furthermore, if $B$ is in $\mathscr{M}$ and $f: B \rightarrow A$ is any function, 
define $g: B \rightarrow F A$ by $g(b)=\left(f(b),\left(f(m b)_{m \in M}\right)\right)$. Then

$$
\begin{aligned}
g(n b) & =\left\{g\left(b^{\prime}\right) \mid b^{\prime} \in n b\right\} \\
& =\left\{\left(f\left(b^{\prime}\right),\left(f\left(m b^{\prime}\right)\right)_{m \in M}\right) \mid b^{\prime} \in n b\right\} \\
& =\left\{\left(f\left(b^{\prime}\right),(f((m n) b))_{m \in M}\right) \mid b^{\prime} \in n b\right\} \\
& =n g(b),
\end{aligned}
$$

because of the $\mu$-condition on $B$, so $g$ is a homomorphism and is easily seen to be unique with the property that $p_{A} g=f$. This proves the following result.

THEOREM 1. If $M$ is a commutative semigroup, the category of all sets equipped with $\mu$-representations of $M$ is cotripleable over the category of sets.

It will follow from Theorem 2 that the word "commutative" is not necessary for the truth of Theorem 1 .

3. Cotheories. The preceding would have gone through with little change if we had taken $m: A \rightarrow P^{2} A=P P A$ and interpreted $m(n a)=(m n) a$ to mean that $a^{\prime} \in \alpha \in n a$ implies $m a^{\prime}=(m n) a$. This suggests the following analogy with the notion of an algebraic theory in universal algebra. Define a cotheory to be a category $T$ with objects $0,1,2, \cdots$, distinguished maps $\pi_{n}^{n+1}: n \rightarrow n+1$, and a functor $P: T \rightarrow T$ where $P(n)=n+1$. An algebra over $T$ is to be a functor $X: T \rightarrow \mathscr{S}$ such that $X(n)=P^{n} A$ for a fixed set $A$, $X P=P X$, and $X\left(\pi_{n}^{n+1}\right)$ takes $\alpha$ to $\{\alpha\}$. We denote $X(\sigma)$ by $\sigma_{A}$ for each $\sigma$ in $T$ and say that $A$ is an algebra. The reasonable condition for a homomorphism $f: A \rightarrow B$ is that for each $\sigma: n \rightarrow m,\left(P^{m} f\right)\left(\sigma_{A}(\alpha)\right)=\sigma_{B}\left(P^{n} f\right)(\alpha)$ where $P$ is covariant (direct image). By $P^{0} A$ we shall mean $A$. If $T$ is generated by maps $0 \rightarrow m$, we shall say that $T$ is standard. This seems to be the correct generalization of multivalued operations, and is of significance since the category $\mathscr{A}$ of algebras over a standard cotheory is easily seen to be right complete, and $U: \mathscr{A} \rightarrow \mathscr{S}$ preserves colimits and satisfies the precise cotripleableness condition. Hence, by the adjoint functor theorem [5], $\mathscr{A}$ is cotripleable iff $U$ satisfies the cosolution set condition.

We have been able to obtain cosolution sets only when an additional strong condition is imposed on $T$. If $n>m$, define $\pi_{m}^{n}=\pi_{n-1}^{n} \circ \cdots \circ \pi_{m}^{m+1}$. Suppose that, whenever $\theta: 0 \rightarrow m$ and $\sigma: 0 \rightarrow n$ in $T$, there is $\theta * \sigma: 0 \rightarrow m$ such that the equation $\left(P^{n} \theta\right) \circ \sigma=\pi_{m}^{m+n} \circ(\theta * \sigma)$ holds in $T$. Such a standard cotheory will be called tractable. Now, for each set $A$, define

$$
F A=A \times \prod_{n=0}^{\infty}\left(P^{n} A\right)^{T(0, n)}
$$


If $\sigma: 0 \rightarrow n$, define

$$
\sigma_{F A}\left(a,\left(\alpha_{\theta}\right)\right)=\left\{\cdots\left\{\left(a^{\prime},\left(\alpha_{\theta * \sigma}\right)\right) \mid a^{\prime} \in \alpha_{1}\right\} \cdots \mid \alpha_{n-1} \in \alpha_{\sigma}\right\} .
$$

Unfortunately the equations in $T$ need not hold in $F A$. However, if $B$ is a $T$-algebra and $f: B \rightarrow A$ is a function, we can define $g: B \rightarrow F A$ by

$$
g(b)=\left(f(b),\left(\left(P^{m} f\right) \theta_{B}(b)\right)_{\theta: 0 \rightarrow m}\right)
$$

and $g$ can be seen to be a homomorphism whose image is fortunately a $T$ subalgebra of $F A$. Hence the set of $T$-subalgebras of $F A$ is a cosolution set for $A$. We have obtained the following generalization of Theorem 1 .

THEOREM 2. The category of algebras over a tractable standard cotheory is cotripleable over $\mathscr{S}$.

\section{REFERENCES}

1. R. C. Davis, Abstract universal algebra, Dissertation, Tulane University, New Orleans, La., 1967.

2. - Quasicotripleable categories, Proc. Amer. Math. Soc. (to appear).

3. J. Duskin, Variations on Back's tripleability criterion, Reports of the Midwest Category Seminar III, Springer, Berlin, 1969, pp. 74-129. MR 40 \#5691.

4. F. Linton, Some aspects of equational categories, Proc. Conf. Categorical Algebra (La Jolla, Calif., 1965), Springer, New York, 1966, pp. 84-94. MR 35 \#233.

5. B. Pareigis, Kategorien und Funktoren, Mathematische Leitfäden, Teubner, Stuttgart, 1969; English transl., Pure and Appl. Math., vol. 39, Academic Press, New York, 1970. MR 42 \#337a,b.

Department of Mathematics, Southern Methodist University, Dallas, Texas 75222 